\title{
Toward Quantification of Ti-Oxidation States in Planetary Materials via Application of the EELS White-Line Ratio Technique
}

\author{
Lindsay R. Slick ${ }^{1}$, Prajkta Mane ${ }^{1}$, Jane Howe ${ }^{1,2}$, Thomas Zega ${ }^{1,3}$ \\ ${ }^{1}$ Lunar and Planetary Laboratory, The University of Arizona, Tucson AZ. \\ ${ }^{2}$ Hitachi High Technologies America, Clarksburg MD. \\ ${ }^{3}$ Materials Science and Engineering, The University of Arizona, Tucson AZ.
}

Electron energy-loss spectroscopy (EELS) is a technique that gives insights into the electronic structure of atoms. Coupled to a transmission electron microscope (TEM), EELS can be used to map a sample at the atomic level and probe for chemical information such as oxidation state, site symmetry, and coordination. For $3 d$ transition metals, e.g., Fe, Ti, this information is stored in electronic transitions from the $2 p$ core shell [1]. Ti is of particular interest in planetary science because of its diverse valency (i.e., $\mathrm{Ti}^{3+}, \mathrm{Ti}^{4+}$ ) in calcium- and aluminum- rich inclusions (CAIs) [2]. Thought to be the oldest formed solids in our solar system $(4.567 \mathrm{Ga})$, the chemical origins of CAIs probe the thermodynamic landscape of the early solar nebula [3]. Our goal is to quantify the Ti oxidation states preserved in materials relevant to the early solar nebula down to the atomic level to gain insights into the redox conditions under which these materials formed or last equilibrated.

The EELS $\mathrm{L}_{2,3}$ absorption edges of $3 d$ transition metals show sensitivity to oxidation state in the form of a chemical shift to higher energies with valence increase [4]. Approaches to quantifying the edges depend on the energy-loss near-edge structure (ELNES). In Fe, the $\mathrm{L}_{2,3}$ edges are Gaussian-shaped, enabling fitting with linear combinations of spectra acquired from end member references [5]. In comparison, Ti can exhibit more complex ELNES, making it challenging to fit the spectra with endmember references. The white-line ratio method is potentially an attractive alternative means of quantifying the Ti $\mathrm{L}_{2,3}$ edges because it tracks chemical shift rather than the ELNES, which is affected by the local crystal field. [6,4].

The white-line ratio approach for $\mathrm{Ti}$ integrates the intensities within two fixed windows, placed at the lowest energy peak of the $\mathrm{L}_{3}$ edge and the highest energy peak of the $\mathrm{L}_{2}$ edge, and relates their ratio, $\mathrm{IL}_{2} / \mathrm{IL}_{3}$, to oxidation state [6]. We note that [4] performed a systematic study of Ti oxides, and reported a calibration curve relating white line intensity ratios to the abundance of $\mathrm{Ti}^{4+}$ in a set of standards. To establish these techniques under our own calibrations, instrumentation, and optical conditions, we also acquired EELS spectra of $\mathrm{Ti}$ oxides of known $\mathrm{Ti}^{3+} / \mathrm{Ti}^{4+}$ content, shown in Table 1 . All spectra were corrected for channel-to-channel gain variation and dark current. An inverse power law ( $\left.\mathrm{AE}^{-1}\right)$ model was used to subtract background, and plural scattering was removed via Fourier-ratio deconvolution. Removal of the intensity beneath the $\mathrm{L}_{2,3}$ edge was performed using a step function described by [6].

The EELS spectra were collected over several days using dispersions from $0.025-0.25 \mathrm{eV} / \mathrm{channel}$. To ensure consistent internal calibration, we utilized an $\mathrm{O} \mathrm{K}$ edge $(532 \mathrm{eV})$ as an internal reference energy. The shift in $\mathrm{O}$ edges with respect to $\mathrm{Ti}$ as reported in [4] are an order of magnitude smaller than the reported Ti shifts, and no shift is expected of $\mathrm{O}$ with respect to additional cations or structure $[7,8]$. Table 1 lists the centroid peaks of each $\mathrm{L}_{2,3}$ edge according to their energy loss for each reference oxide. As expected, the energy loss of the peaks increases with increasing amounts of $\mathrm{Ti}^{4+}$. Figure 1 shows the $\mathrm{IL}_{2} / \mathrm{IL}_{3}$ determined for each of the reference materials. The integrated $\mathrm{IL}_{2} / \mathrm{IL}_{3}$ ratio ranges from 4.616 $\left(\mathrm{Ti}_{2} \mathrm{O}_{3}\right)$ to $64.250\left(\mathrm{TiO}_{2}\right)$. The data are shown with an exponential curve fit as a function of $\mathrm{Ti}^{4+}$, although 
errors have yet to be calculated. Additionally, we plan to add data from a mixed-valence $\mathrm{Ti}_{4} \mathrm{O}_{7}$ to better constrain the fit.

To test the efficacy of this calibration, we applied the curve to two synthetic $\mathrm{CaTiO}_{3}$ perovskites (CTO) purchased from a commercial supplier (purity level 99+\%). Stoichiometrically, the as-received CTO (38 $\mathrm{wt} \% \mathrm{Ti}$ ) is composed entirely of $\mathrm{Ti}^{4+}$, and so the $\mathrm{IL}_{2} / \mathrm{IL}_{3}$ should be similar to that of $\mathrm{TiO}_{2}$ (rutile). However, our $\mathrm{IL}_{2} / \mathrm{IL}_{3}$ measurements correspond to a $\% \mathrm{Ti}^{4+}$ of $108.9 \%$ ("Synthetic1") and $180.3 \%$ (Synthetic2") placing them above our $100 \% \mathrm{Ti}^{4+}$ standard. In comparison, we measured a CTO sample that was heated under reducing conditions $\left(1200^{\circ} \mathrm{C} \log f \mathrm{O}_{2}=-10\right.$ bars, 2 hours) with the goal of reducing some of the Ti. Our EELS measurements revealed a $\% \mathrm{Ti}^{4+}$ of approximately 0.841 , suggesting the heated sample was successfully reduced, which we hypothesize is via the creation of oxygen vacancies.

Our first order fit of three reference oxides matches literature in shape, but our parameters vary significantly from [4]. We suspect that the addition of $\mathrm{Ti}_{4} \mathrm{O}_{7}$, the averaging of multiple spectral peak identifications, and error calculation will improve the fit, as well as reconcile the discrepancy between the $\mathrm{IL}_{2} / \mathrm{IL}_{3}$ of $\mathrm{TiO}_{2}$ and our synthetic perovskites. Once these measurements are complete, we plan to assess perovskites found in meteorites dating back some 4.567 Ga [9].

\section{References:}

[1] L.A.J, Garvie, A.J. Crvaven, and R Brydson, Amer. Mineralogist 79 (1994), p.411-425.

[2] J.R. Beckett, et al. Geochem. Cosmochem. Acta 52 (1988), p.1479-1495.

[3] J.N. Connelly, et al. Science 338 (2012), p.651-655.

[4] E. Stoyanov, F. Langenhorst, and G. Steinle-Neimann, Amer. Mineralogist 92 (2007), p.557-586.

[5] T.J. Zega, L.A.J. Garvie, and P.R. Buseck. Amer.Minerologist 88 (2003), p.1169-1172.

[6] P.A. Van Aken and B. Liebscher, Phys Chem Minerals 29 (2002), p.188-200.

[7] Z. Wu et al, J. Synchrotron Rad. 8 (2001), p.934-936.

[8] R. Brydson et al, J. Phys.: Condens. Matter 1 (1989), p.797-812.

[9] We gratefully acknowledge NASA (grants \#NNX12AL47G and \#NNX15AJ22G) and NSF (grants \#1531243 and \#0619599) for funding of instrumentation in the Kuiper Materials Imaging and Characterization Facility at the Lunar and Planetary Laboratory, University of Arizona

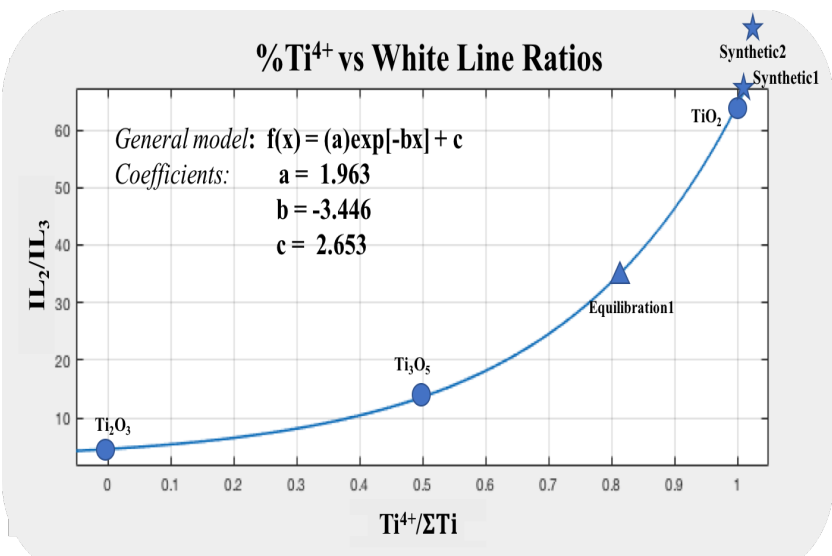

\begin{tabular}{|c|c|c|c|}
\hline Oxide & $\mathbf{T i}^{\mathbf{3 +}}: \mathbf{T I}^{\mathbf{4 +}}$ & $\mathbf{L}_{\mathbf{3}}$ peaks $(\mathbf{e V})$ & $\mathbf{L}_{\mathbf{2}}$ Peaks $(\mathbf{e V})$ \\
\hline $\mathbf{T i O}$ & $0: 0$ & 456.28 & 461.53 \\
\hline $\mathbf{T i}_{\mathbf{2}} \mathbf{O}_{\mathbf{3}}$ & $1: 0$ & 459.368 & 464.368 \\
\hline $\mathbf{T i}_{\mathbf{3}} \mathbf{O}_{\mathbf{5}}$ & $2: 1$ & 459.71 & 464.96 \\
\hline $\mathbf{T i O}_{\mathbf{2}}$ & $0: 1$ & $458.05,460.55$ & $463.55,465.55$ \\
\hline
\end{tabular}

Table 1. Peak positions for oxide standards, listed from least to most $\mathrm{Ti}^{4+}$.

Fig 1. Calibration curve relating the white-line intensity ratio $\left(\mathrm{IL}_{2} / \mathrm{IL}_{3}\right)$ as a function of $\mathrm{Ti}^{4+}$ abundance in a set of reference materials. The exponential fit parameters are inset. 